\title{
Effect of leukocyte alteration on treatment outcomes following preoperative chemoradiotherapy in patients with rectal cancer
}

Tae Gyu Kim, MD ${ }^{1,2}$, Won Park, MD², Doo Ho Choi, MD², Hee Chul Park, MD², Seok-Hyung Kim, MD²,

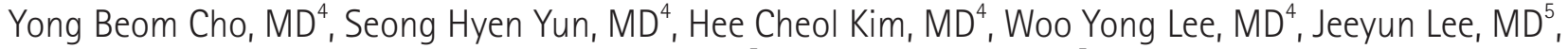
Joon Oh Park, MD ${ }^{5}$, Young Suk Park, MD

'Department of Radiation Oncology, Samsung Changwon Hospital, Sungkyunkwan University School of Medicine, Changwon; Departments of ${ }^{2}$ Radiation Oncology, ${ }^{3}$ Pathology, ${ }^{4}$ Surgery, and ${ }^{5}$ Internal Medicine, Samsung Medical Center, Sungkyunkwan University School of Medicine, Seoul, Korea

Purpose: Hematotoxicity following anti-cancer treatment is known to be related to treatment efficacy in several malignancies. The purpose of this study was to examine the hematologic parameters related to the tumor response and survival in patients treated with curative surgery following preoperative chemoradiotherapy (CRT) for rectal cancer.

Materials and Methods: Four hundred eighteen patients with rectal cancer who underwent preoperative CRT and curative surgery were analyzed, retrospectively. The main clinical factors and blood cell counts before and after CRT were investigated with respect to their relationships with tumor downstaging and patient survival.

Results: The post-CRT leukocyte count was significantly different between the tumor downstaging group and the nondownstaging group (median, 4740/uL vs. 5130/uL; $p=0.013$ ). Multivariate analysis showed that histological grade, circumferential extent, and post-CRT leukocyte count were related to tumor downstaging. In addition, histological grade, post-CRT leukocyte count, and tumor downstaging were related to disease-free survival. The 5 -year disease-free survival and overall survival in patients with post-CRT leukocyte count $\leq 3730 / \mathrm{LL}$, which is the cut-off value derived from the receiver operation characteristic (ROC) curve analysis, were significantly higher than those with higher counts ( $88.0 \%$ vs. $71.6 \%, p=0.001 ; 94.4 \%$ vs. $84.1 \%, p=0.024)$.

Conclusion: Post-CRT leukocyte count of $\leq 3730 / \mathrm{uL}$ could be regarded as a good prognostic factor for tumor response and survival in rectal cancer patients treated with preoperative CRT.

Keywords: Rectal neoplasms, Chemoradiotherapy, Leukopenia, Radiation effects

\section{Introduction}

Hematologic toxicity such as neutropenia and leukopenia during or after chemotherapy has been reported to be a useful independent biomarker for predicting survival in patients with cancers such as non-small cell lung cancer, gastric cancer, breast cancer, Hodgkin's disease, and colorectal cancer [1-6]. In a retrospective study of metastatic colorectal cancer, patients

Received 20 May 2017, Revised 28 June 2017, Accepted 3 July 2017.

Correspondence: Won Park, MD, Department of Radiation Oncology, Samsung Medical Center, Sungkyunkwan University School of Medicine, 81 Irwon-ro, Gangnam-gu, Seoul 06351, Korea. Tel: +82-2-3410-2616, Fax: +82-2-3410-2619, E-mail: wonro.park@samsung.com

(c) This is an Open Access article distributed under the terms of the Creative Commons Attribution Non-Commercial License (http://creativecommons.org/ licenses/by-nc/4.0/) which permits unrestricted non-commercial use, distribution, and reproduction in any medium, provided the original work is properly cited.

www.e-roj.org 
with severe neutropenia during chemotherapy had a median survival of 26 months, while those without neutropenia had a median survival of 13.6 months [7].

In addition to hematologic toxicity, several hematologic parameters such as hemoglobin level, the proportion of lymphocytes among white blood cells (WBC), and the ratio of neutrophil to lymphocyte have been reported to be related to treatment efficacy in rectal cancer patients. In several retrospective studies, high hemoglobin levels before and during chemoradiotherapy (CRT) for rectal cancer had a relationship with improved tumor response and survival $[8,9]$. Other studies have suggested that rectal cancer patients with higher proportions of lymphocytes among their WBCs and lower neutrophil to lymphocyte ratios had more favorable survival rates than those without these characteristics $[10,11]$.

In this study, we retrospectively evaluated the alteration of hematologic parameters during neoadjuvant chemoradiotherapy in patients with rectal cancer and found useful hematologic parameters for predicting treatment efficacy, including tumor response and survival.

\section{Materials and Methods}

\section{Patients}

Neoadjuvant concurrent CRT was performed in 507 patients who had locally advanced rectal cancer or distal rectal cancer for which an abdominoperineal resection is thought to be necessary, at Samsung Medical Center between July 1999 and March 2009. Of the 507 patients, 31 who had distant metastasis at the time of first diagnosis, 18 who did not undergo curative rectal surgery, and 40 who received reduced chemotherapy for poor performance status were excluded from this investigation. The remaining 418 patients with rectal cancer were analyzed in terms of tumor downstaging and survival.

Also, additional 212 patients treated from April 2009 to December 2010 were evaluated to verify the impact of postCRT leukocyte count on tumor downstaging and tumor regression grade (TRG) suggested by Mandard et al. [12]. Of these, 8 had distant metastasis at initial staging work ups, 11 underwent reduced chemotherapy, 8 underwent S-1 chemotherapy, and 17 did not receive curative surgery. Remaining 168 patients were analyzed for verification.

All patients underwent digital rectal examination, chest $X$-ray, colonoscopy, and abdominal and pelvic computed tomography (CT) scans before beginning neoadjuvant CRT. Of them, 227 patients (54.3\%) underwent pelvic magnetic resonance imaging scans as staging work ups. In all patients, complete blood cell counts (CBC) including hemoglobin, leukocyte, and leukocyte subsets were evaluated within 2 weeks before the start of both CRT and surgery. Informed consent for the treatments was collected from all patients, and this study was approved by the Institutional Review Board of Samsung Medical Center (IRB No. SMC201410134).

\section{Treatment}

The radiation dose was a total of 44-45 Gy with 1.8-2 Gy per fraction, using one posterior beam and two bilateral beams with 4-10 megavoltage photons. The radiation field was the whole pelvis, including the primary tumor and the regional Iymphatic area with customized portals. In all patients, chemotherapy was delivered concurrently with radiotherapy (RT) consisting of a bolus injection of 5-fluorouracil, $500 \mathrm{mg} /$ $\mathrm{m} 2 /$ day for 3 days per cycle for the first and last week of RT or capecitabine, $850 \mathrm{mg} / \mathrm{m}^{2} /$ day twice daily for 5 days per week for 5 weeks. Adjuvant chemotherapy was administered to all but 88 of the patients. All of the subjects underwent curative surgery 6-8 weeks after the end of the CRT, including low anterior resection in 357 patients, abdominopelvic resection in 80 patients, and local excision in 21 patients. After surgery, all tumor specimens were examined by a pathologist, and the post-CRT pathologic stage was evaluated. Well or moderatelydifferentiated tumors were classified as low grade tumor, and poorly differentiated tumor, mucinous carcinoma or signet ring cell carcinoma were classified as high grade tumor $[13,14]$. The TNM stage system according to the 7th edition of the American Joint Committee on Cancer was used in staging. Tumor downstaging was determined by comparing the pretreatment clinical and the postoperative pathological $T$ and $\mathrm{N}$ classifications and defined as lower pathological stage than pretreatment stage. For example, patients with clinical stage III and pathological stage III were classified as non-downstaging group, while patients with clinical stage III and pathological stage II were classified as downstaging group.

\section{Follow-up and assessment of disease recurrence}

Patient follow-ups by a colorectal surgeon, radiation oncologist, or medical oncologist were performed every 3 months for the first 2 postoperative years and every 6 months thereafter. Physical examination, digital rectal examination, complete blood count, liver function test, and the carcinoembryonic antigen (CEA) value assessment were performed as follow-up evaluations. Chest radiography and CT of the abdomen and pelvis were conducted every 6 
Table 1. Patient's characteristics according to the tumor response after concurrent chemotherapy in preoperative rectal cancer patients

\begin{tabular}{|c|c|c|c|}
\hline Variable & Downstaging group & Non-downstaging group & $p$-value \\
\hline Gender & & & 0.685 \\
\hline Male & $146(67.9)$ & $133(65.5)$ & \\
\hline Female & $69(32.1)$ & $70(34.5)$ & \\
\hline Age (yr) & & & 0.762 \\
\hline$<60$ & $132(61.4)$ & $121(59.6)$ & \\
\hline$\geq 60$ & $83(38.6)$ & $82(40.4)$ & \\
\hline Distance from anal verge $(\mathrm{cm})$ & & & 0.686 \\
\hline$<4$ & $72(33.5)$ & $64(31.5)$ & \\
\hline$\geq 4$ & $143(66.5)$ & $139(68.5)$ & \\
\hline TNM stage & & & 0.602 \\
\hline I & $15(7.0)$ & $11(5.4)$ & \\
\hline ॥ & $58(27.0)$ & $49(24.1)$ & \\
\hline III & $142(66.0)$ & $143(70.4)$ & \\
\hline cT & & & $0.001^{*}$ \\
\hline cT1 & $2(0.9)$ & $2(1.0)$ & \\
\hline cT2 & $18(8.4)$ & $14(6.9)$ & \\
\hline cT3 & $163(75.8)$ & 179 (88.2) & \\
\hline cT4 & $32(14.9)$ & $8(3.9)$ & \\
\hline $\mathrm{cN}$ & & & 0.308 \\
\hline $\mathrm{cNO}$ & $75(34.9)$ & $61(30.0)$ & \\
\hline $\mathrm{cN}+$ & $140(65.1)$ & $142(70.0)$ & \\
\hline Histological grade & & & $0.002^{*}$ \\
\hline Low & $202(94.0)$ & $172(84.7)$ & \\
\hline High & $13(6.0)$ & $31(15.3)$ & \\
\hline Pretreatment tumor size $(\mathrm{cm})$ & & & 0.262 \\
\hline$\leq 5$ & $145(67.4)$ & $126(62.1)$ & \\
\hline$>5$ & 70 (32.6) & 77 (37.9) & \\
\hline Circumferential extent (\%) & & & $0.014^{*}$ \\
\hline$\leq 60$ & $103(47.9)$ & $72(35.5)$ & \\
\hline$>60$ & $112(52.1)$ & $131(64.5)$ & \\
\hline Pretreatment CEA level (ng/mL) & & & $0.035^{*}$ \\
\hline$\leq 5$ & $153(71.2)$ & $132(65.0)$ & \\
\hline$>5$ & $44(20.5)$ & $62(30.5)$ & \\
\hline Unknown & $18(8.4)$ & $9(4.4)$ & \\
\hline Chemotherapy regimen & & & 0.368 \\
\hline 5-FU & $134(62.3)$ & $136(67.0)$ & \\
\hline Capecitabine & $81(37.7)$ & & \\
\hline
\end{tabular}

Values are presented as number (\%).

CEA, carcinoembryonic antigen; FU, fluorouracil.

${ }^{*} p<0.05$.

months after treatment, and colonoscopy or sigmoidoscopy were performed every year after surgery. Suspected lesions of recurrence were proven pathologically by biopsy and/or clinically by close follow-up imaging showing an increase in size over time.

\section{Statistical analyses}

The enrolled patients were divided into two groups: the downstaging group (DG) and the non-downstaging group
(NG). The patient and tumor characteristics between $D G$ and NG were compared using the chi-square test or Fisher exact test for categorical variables and the Mann-Whitney U-test for continuous variables. The alterations of blood test data between the pre-CRT and post-CRT phases were evaluated using the Wilcoxon signed-rank test. A receiver operating characteristic (ROC) curve was used to define the cut-off point for the continuous variable that was related to tumor downstaging. Multivariate stepwise logistic regression analysis 


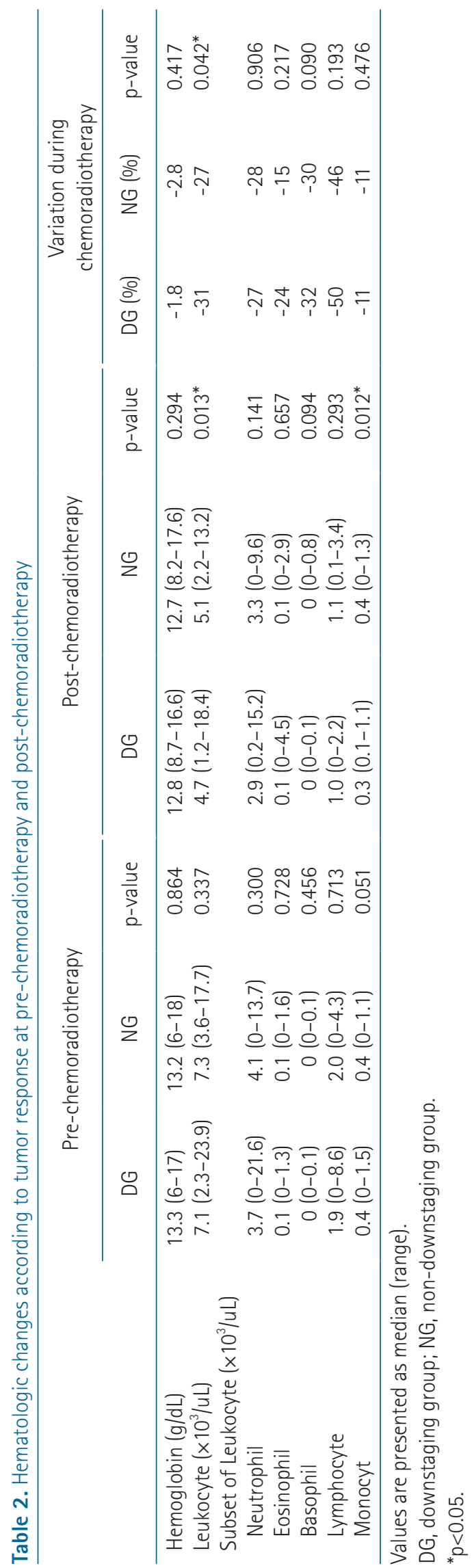

was used to determine the correlations between main potential parameters and tumor downstaging. The disease-free survival (DFS) curves and overall survival (OS) curves according to main potential parameters were estimated using the KaplanMeier method. The difference between the survival curves were evaluated in a univariate manner with the log-rank test and in a multivariate manner with the Cox's proportional hazards model. All statistical analyses were performed using SPSS software ver. 20.0 (IBM, Armonk, NY, USA). A two-sided $p$-value of $<0.05$ was considered statistically significant.

\section{Results}

\section{Patient characteristics}

The median follow-up period was 52 months (range, 5 to 138 months). The DG and NG included 215 patients and 203, respectively. The patient characteristics of the two groups are shown in Table 1. The main clinical and pathological factors did not significantly differ between the two groups, with the exception of clinical T stage, the histological grade, circumferential extent, and pretreatment CEA level. The patients in DG had more tumors with low grade and short circumferential extent and showed lower pretreatment CEA level than the patients in NG.

\section{Blood cell data}

The blood cell data was evaluated before and after the CRT. The second blood test was performed on median 39 days (range, 28 to 55 days) after the end of CRT. The data variation over time did not differ between the DG and NG, except for the post-CRT leukocyte count, post-CRT monocyte count, and the reduction ratio of leukocytes (Table 2). The post-CRT leukocyte count had a median of 4740/uL (range, 1200/uL to 18370/uL) in DG and a median of 5130/uL (range, 2210/uL to 13220/uL) in NG ( $p=0.013)$ (Fig. 1). The reduction ratio of leukocyte count had a median of $-31 \%$ (range, $-83 \%$ to $134 \%$ ) and a median of $-26.5 \%$ (range, $-71 \%$ to $-62 \%$ ) in the DG and NG groups, respectively $(p=0.042)$. The post-CRT monocyte counts were lower in DG than in NG, significantly (median, 395/uL and 439/ $u L$, respectively; $p=0.012$ ). However, the reduction ratio of monocyte counts were not different between the two groups ( $p$ $=0.476$ ).

The leukocyte count and the percent of lymphocyte were significantly reduced from pre-CRT to post-CRT in both $D G$ and $N G(p<0.001)$. On the other hand, the percent of neutrophils and the ratio of neutrophils to lymphocytes were significantly increased between the two time periods irrespective of the 


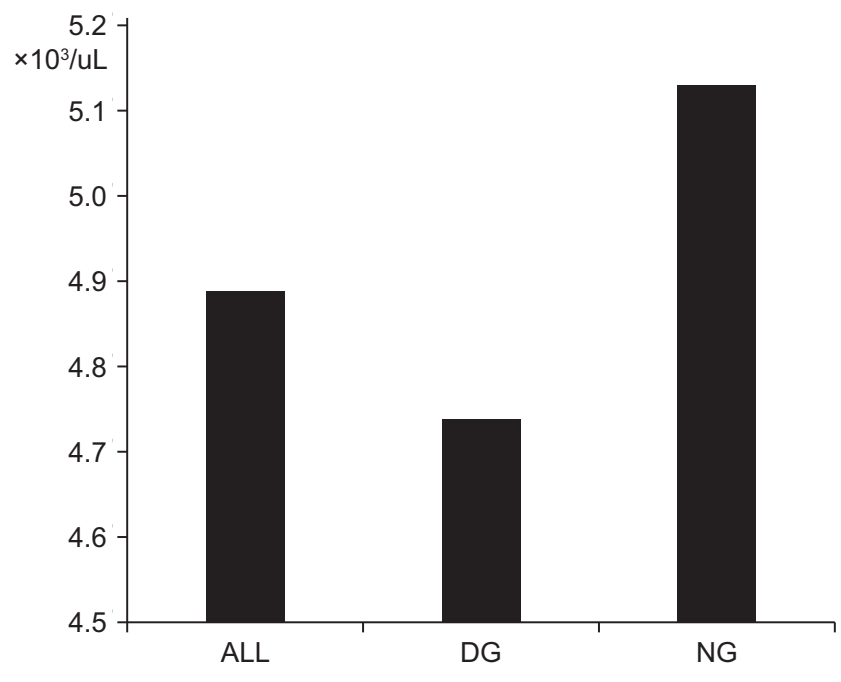

Fig. 1. Median post-CRT leukocyte counts. CRT, chemoradiotherapy; DG, downstaging group; NG, non-downstaging group.

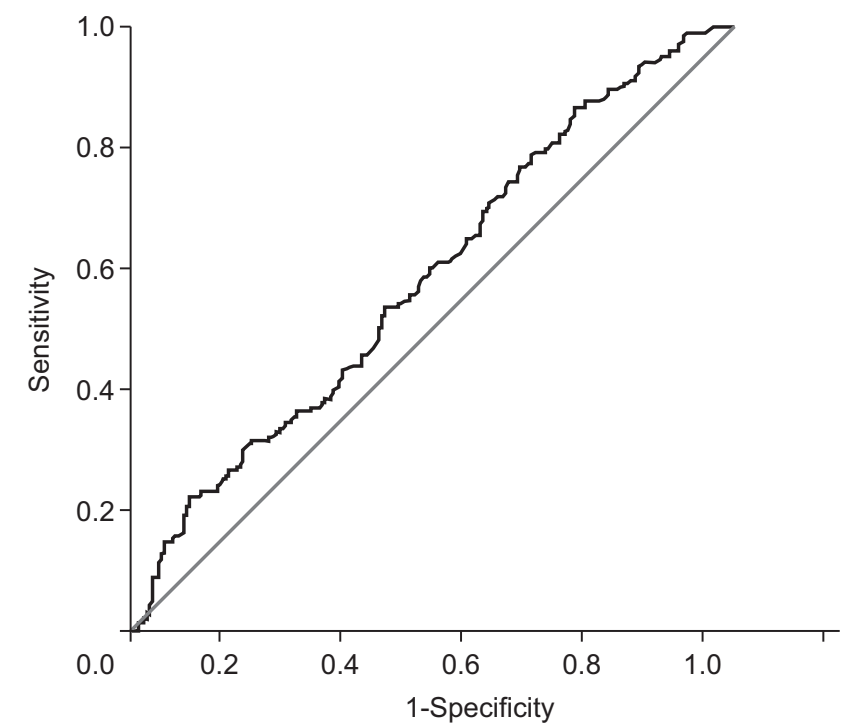

Fig. 2. A receiver operating characteristic (ROC) curve of postCRT leukocyte counts in predicting the tumor downstaging. CRT, chemoradiotherapy.

Table 3. Multivariate analysis for tumor downstaging

\begin{tabular}{|c|c|c|}
\hline Variable & OR (95\% Cl) & p-value \\
\hline Gender (male vs. female) & $1.391(0.880-2.199)$ & 0.165 \\
\hline Age $>60$ yr ( $\leq 60$ vs. $>60)$ & $0.946(0.615-1.456)$ & 0.806 \\
\hline Distance from anal verge $(\leq 4 \mathrm{~cm}$ vs. $>4 \mathrm{~cm})$ & $1.082(0.679-1.723)$ & 0.743 \\
\hline \multicolumn{3}{|l|}{ Clinical TNM stage } \\
\hline (I vs. II) & $0.742(0.294-1.874)$ & 0.535 \\
\hline (I vs. III) & $1.124(0.685-1.844)$ & 0.649 \\
\hline Histological grade (low grade vs. high grade) & $3.139(1.467-6.720)$ & $0.003^{*}$ \\
\hline Circumferential extent ( $\leq 60 \%$ vs. >60\%) & $1.618(1.042-2.513)$ & $0.039^{*}$ \\
\hline Pretreatment CEA level ( $\leq 5 \mathrm{ng} / \mathrm{mL}$ vs. $>5 \mathrm{ng} / \mathrm{mL}$ ) & $1.405(0.871-2.266)$ & 0.160 \\
\hline Chemotherapy regimen (5-FU vs. capecitabine) & $0.771(0.500-1.188)$ & 0.248 \\
\hline Post-CRT leukocyte count ( $\leq 3730 / u L$ vs. $>3730 / u L)$ & $2.698(1.547-4.706)$ & $<0.001^{*}$ \\
\hline
\end{tabular}

$\mathrm{OR}$, odds ratio; $\mathrm{Cl}$, confidence interval; $\mathrm{CEA}$, carcinoembryonic antigen; FU, fluorouracil; $\mathrm{CRT}$, chemoradiotherapy. ${ }^{*} p<0.05$.

incidence of downstaging $(p<0.001)$.

\section{Predictive factors for tumor downstaging}

The major clinical and hematological parameters which showed a significant difference between the DG and NG were analyzed using a multivariate model (Table 3). The cut-off post-CRT leukocyte value derived from ROC curve analysis for predicting the downstaging was 3730/uL, and all of the patients were divided into two groups based on the cut-off value ( $p=0.003 ; A U C=0.583 ;$ Youden's index $=0.133 ; 95 \%$ confidence interval, 0.534-0.631) (Fig. 2). The histological grade, tumor circumferential extent, and post-CRT leukocyte value were independently related to tumor downstaging. There was a particularly favorable rate of downstaging, with an odds ratio of 2.698 , with post-CRT leukocyte count of $\leq 3730 / \mathrm{uL}$, as compared to cases where counts were $>3730 / \mathrm{uL}$.

\section{Prognostic factors for patient survival}

Table 4 showed the prognostic factors for patient diseasefree survival and overall survivial. Histological grade, circumferential extent of tumor, pretreatment CEA level, postCRT leukocyte count, and tumor downstaging were related to DFS in univariate analyses. Multivariate analyses showed that histological grade, post-CRT leukocyte count, and tumor 

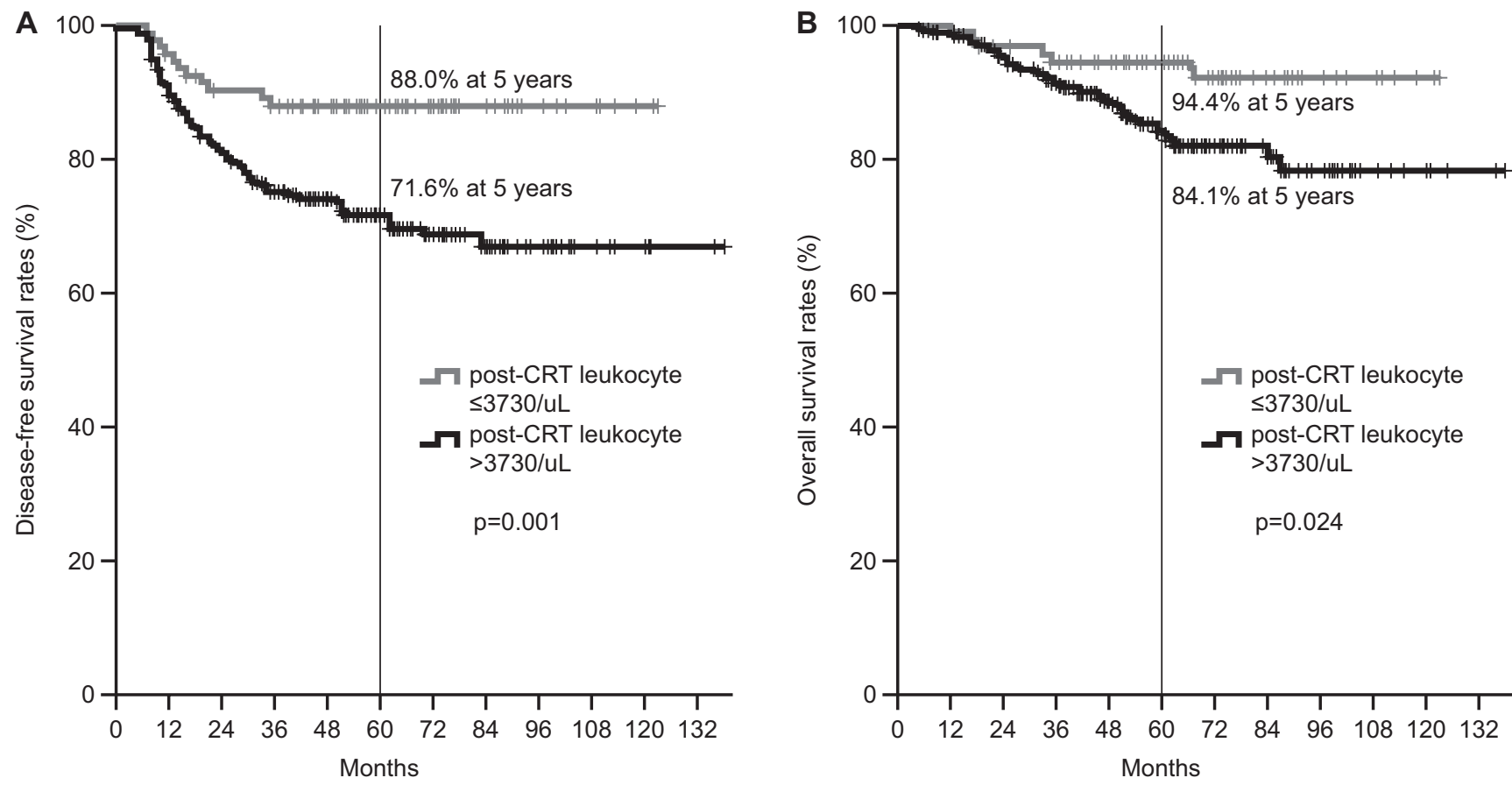

Fig. 3. Disease-free survival (A) and overall survival (B) curves according to the post-CRT leukocyte count. The patients with lower post-CRT leukocytes had significantly more favorable disease-free survival and overall survival than those with higher post-CRT leukocyte counts. CRT, chemoradiotherapy.

downstaging were independent factors $(p=0.042,0.003$, and 0.001 , respectively). The 5 -year DFS rate was $88.0 \%$ in patients with post-CRT leukocyte counts of $\leq 3730 / \mathrm{uL}$ and $71.6 \%$ in patients with higher counts (Fig. 3A).

The histological grade, circumferential extent, chemotherapy regimen, post-CRT leukocyte count, and tumor downstaging showed significant correlation with $0 S$ rate. Multivariate analyses for OS revealed that a high grade of tumor was an independent poor prognostic factor $(p=0.001)$. The 5 -year OS rate was high in patients with post-CRT leukocyte counts of $\leq 3730 / \mathrm{uL}$, compared with patients with higher counts $(94.4 \%$ vs. $84.1 \% ; p=0.024$ ) (Fig. 3B).

\section{Verification of relationship between post-CRT leukocyte count and tumor response}

In the verification analysis of additional 168 patients, the impact of post-CRT leukocyte count on tumor response was verified. The lower post-CRT leukocyte group with the count of $\leq 3730 /$ L had more tumor downstaging than the higher group, significantly (77.1\% vs. 57.1\%; $p=0.026$ ) (Table 5). There was no significant difference of TRG between the two groups, though the lower leukocyte group had tendency of having higher TRG.

\section{Discussion and Conclusion}

CBC count is tested in an inexpensive, reliable, reproducible, and standardized manner and is a biological marker proven to be a prognostic factor for several systemic and solid malignancies. Numerous studies showed that pretreatment $\mathrm{CBC}$ count and its components could be prognostic tools in various type of tumors with some being used as prognostic score components-e.g. leukocyte count in the International Prognostic Score (IPS) of Hodgkin's Iymphoma [15]. In addition to leukocyte count, the absolute neutrophil count, the neutrophil to lymphocyte ratio, the absolute lymphocyte count, the absolute monocyte count, the hemoglobin concentration and the platelet count have been suggested to be prognostic factors in particular malignancies [16-22].

There are several reports that the post-treatment $\mathrm{CBC}$ count or hematotoxicity following chemotherapy, radiotherapy, and immunotherapy are related to treatment efficacy including tumor response and patient survival. Leukopenia following chemotherapy was revealed to be related with patient survival in studies of breast cancer. Saarto et al. [23] demonstrated that patients with a lower leukocyte nadir during chemotherapy for node-positive stage II and III breast cancer had significantly better disease-free and 0 rates $(p=0.006$ and $p=0.04$, 
Table 4. Univariate and multivariate analysis with prognostic factors for survivals

\begin{tabular}{|c|c|c|c|c|c|c|c|}
\hline Variable & $\begin{array}{c}\text { No. of } \\
\text { patients }\end{array}$ & $\begin{array}{c}5-y r \text { DFS } \\
(\%)\end{array}$ & Univariate & $\begin{array}{l}\text { Multi- } \\
\text { variate }\end{array}$ & $\begin{array}{c}5-y r \text { OS } \\
(\%)\end{array}$ & Univariate & $\begin{array}{l}\text { Multi- } \\
\text { variate }\end{array}$ \\
\hline Gender & & & 0.969 & 0.564 & & 0.670 & 0.667 \\
\hline Male & 279 & 75.1 & & & 86.1 & & \\
\hline Female & 139 & 75.1 & & & 85.5 & & \\
\hline Age (yr) & & & 0.751 & 0.543 & & 0.085 & 0.220 \\
\hline$\leq 60$ & 253 & 76.1 & & & 88.0 & & \\
\hline$>60$ & 165 & 73.2 & & & 82.2 & & \\
\hline Distance from anal verge $(\mathrm{cm})$ & & & 0.133 & 0.185 & & 0.553 & 0.435 \\
\hline$\leq 4$ & 136 & 71.0 & & & 87.7 & & \\
\hline$>4$ & 282 & 77.1 & & & 85.0 & & \\
\hline Clinical TNM stage & & & 0.547 & 0.785 & & 0.634 & 0.779 \\
\hline 1 & 26 & 84.1 & & & 90.8 & & \\
\hline ॥ & 107 & 77.3 & & & 87.6 & & \\
\hline III & 285 & 73.6 & & & 84.6 & & \\
\hline Histological grade & & & $0.004^{*}$ & $0.042^{*}$ & & $<0.001^{*}$ & $0.001^{*}$ \\
\hline Low & 374 & 77.2 & & & 88.8 & & \\
\hline High & 44 & 57.5 & & & 61.7 & & \\
\hline Circumferential extent (\%) & & & $0.028^{*}$ & 0.138 & & $0.026^{*}$ & 0.113 \\
\hline$\leq 60$ & 175 & 81.0 & & & 90.5 & & \\
\hline$>60$ & 243 & 70.8 & & & 82.3 & & \\
\hline Pretreatment CEA (ng/mL) & & & $0.031^{*}$ & 0.366 & & 0.081 & 0.300 \\
\hline$\leq 5$ & 285 & 77.6 & & & 86.8 & & \\
\hline$>5$ & 106 & 68.9 & & & 83.3 & & \\
\hline Chemotherapy regimen & & & 0.065 & 0.138 & & $0.023^{*}$ & 0.068 \\
\hline 5-FU & 270 & 72.7 & & & 82.4 & & \\
\hline Capecitabine & 148 & 79.5 & & & 90.6 & & \\
\hline Post-CRT leukocyte count (/uL) & & & $0.001^{*}$ & $0.003^{*}$ & & $0.024^{*}$ & 0.152 \\
\hline$\leq 3730$ & 84 & 88.0 & & & 94.4 & & \\
\hline$>3730$ & 334 & 71.6 & & & 84.1 & & \\
\hline Tumor downstaging & & & $<0.001^{*}$ & $0.001^{*}$ & & $0.008^{*}$ & 0.096 \\
\hline Downstaging & 215 & 84.7 & & & 91.5 & & \\
\hline Non-downstaging & 203 & 65.0 & & & 79.5 & & \\
\hline
\end{tabular}

DFS, disease-free survival; OS, overall survival; CEA, carcinoembryonic antigen; CRT, chemoradiotherapy. ${ }^{*} p<0.05$.

respectively). Median DFS and OS time for leukocyte nadirs $\geq 4000,3000-3900$, and $<3000$ were 80 and 116 months, 90 and 111 months, and 126 and 128 months, respectively. The authors suggested that the leukocyte nadir could be used as a marker for therapy efficacy and as tools for establishing an optimal chemotherapy dose intensity for individual patients. Poikonen et al. [4] also showed that a low leukocyte nadir was related to a long DFS in breast cancer patients receiving adjuvant chemotherapy in univariate analyses, though not in multivariate analyses. A German Hodgkin Study Group demonstrated a correlation between severe leukopenia during chemotherapy and reduced treatment failure in 4,626 Hodgkin's disease patients [5]. At a median follow-up time of 66 months, the female patients had a higher prevalence of severe leukopenia and showed a better failure-free survival rate than male patients (69.9\% vs. $55.2 \%$, and $81 \%$ vs. $74 \%$, respectively). Severe leukopenia was associated with better overall survival in both males and females.

Of the different leukocyte subsets, neutropenia during anticancer treatment was proposed to be a prognostic marker in various cancer types. In a pooled analysis of 1,265 patients with advanced non-small cell lung cancer, the hazard ratios of death were 0.65 for patients with severe neutropenia and 0.74 for those with mild neutropenia during chemotherapy [24]. Shitara et al. [7] retrospectively analyzed 153 patients with metastatic colorectal cancer who received FOLFOX as first-line chemotherapy. Of these patients, severe neutropenia during chemotherapy occurred in 46 patients (30\%) and mild neutropenia occurred in 60 patients (39\%), with hazard ratios of deaths of 0.35 and 0.55 , respectively. 
Table 5. Verification of relationship between post-CRT leukocyte count and tumor response

\begin{tabular}{|c|c|c|c|}
\hline \multirow{2}{*}{ Variable } & \multicolumn{2}{|c|}{$\begin{array}{l}\text { Post-CRT leukocyte } \\
\text { count }\end{array}$} & \multirow{2}{*}{ p-value } \\
\hline & $\begin{array}{l}\leq 3730 / u L \\
(n=35)\end{array}$ & $\begin{array}{l}>3730 / \mathrm{uL} \\
(n=133)\end{array}$ & \\
\hline Tumor downstaging & & & $0.026^{*}$ \\
\hline Downstaging & $27(77.1)$ & $76(57.1)$ & \\
\hline Non-downstaging & $8(22.9)$ & $57(42.9)$ & \\
\hline Tumor regression grade & & & 0.394 \\
\hline 0 & $0(0)$ & $3(2.3)$ & \\
\hline 1 & $2(5.7)$ & $14(10.5)$ & \\
\hline 2 & $14(40.0)$ & $61(45.9)$ & \\
\hline 3 & $9(25.7)$ & $20(15.0)$ & \\
\hline 4 & $10(28.6)$ & 35 (26.3) & \\
\hline
\end{tabular}

Values are presented as number (\%).

CRT, chemoradiotherapy.

${ }^{*} \mathrm{p}<0.05$.

In our study, low post-CRT leukocyte count was related to favorable tumor response with the cut-off point established to be $3730 / u L$, lower than the definition value of leukopenia. Interestingly, post-CRT leukocyte count was related to not only tumor downstaging, but also disease-free survival independent of tumor downstaging. Low post-CRT leukocyte count was also found to be related to treatment outcome in breast cancer and Hodgkin's disease, irrespective of clinical stage or the type of chemotherapy concurrently used with the RT. It is unclear whether leukopenia is only a secondary phenomenon of other prognostic factors like tumor extent or fundamental mechanistic events reflecting the patient's survival. Considering that the alteration of leukocytes and neutrophils occur in patients with early stage gastric cancer and uterine cervical intraepithelial neoplasm, the role of leukopenia in predicting the oncologic outcome cannot be excluded $[25,26]$.

According to our results, post-CRT leukopenia might be a clinically applicable therapeutic determinant to adjust doseintensity of adjuvant chemotherapy. Patients with post-CRT leukopenia and a good tumor response may expect a better oncologic outcomes by toxicity based dose reduction, which might reduce expected hematotoxicity and preserve tumor control. On the other hand, the outcome of patients who do not experience leukopenia might be improved by doseescalation of adjuvant chemotherapy.

Leukocytes supplement using nutritional support or granulocyte colony stimulating factor (G-CSF) are considered when severe leukopenia develop during or after CRT. G-CSF treatment could increase the $0 S$ rate by reducing the severity and duration of neutropenic complications while maintaining the prescribed chemotherapy dose intensity [27]. However, G-CSF therapy may promote tumor growth in certain malignancies and may also increase the probability of developing secondary malignancies [28]. Further studies are needed to determine the effect of leukocyte supplementation on tumor response and survival in patients with leukopenia following CRT for rectal cancer.

The possible mechanism behind the correlation between leukopenia during anti-cancer therapy and a favorable oncologic outcome could be explained by two theories. First the accessibility of therapeutic agents to the cancer cell might be similar in normal tissue cells, including the blood stem cells in bone marrow that produce the blood cell precursors because each individual has the same microvascular environment in both normal and tumor tissue. Second, there might be an inherent sensitivity in cancer cells to the anticancer agent. Supposing the sensitivity of cancer cells to anticancer therapy is based on genetic predisposition, this would be the same in an individual's normal tissue cells. As far as the authors know, it is not known which treatment of concurrent chemoradiotherapy for pelvic malignancy is the primary cause of leukopenia.

This is the first report to show that leukopenia is associated with tumor downstaging and favorable survival in rectal cancer patients treated with preoperative CRT. However, first limitation to this study is that, although the CBC was collected within 2 weeks before both the start of CRT and surgery, the collection points differed among the enrolled patients, a consequence of the retrospective design of this study. Second limitation is that tumor downstaging was determined by comparing the clinical and the pathological stage, which may result in inaccuracy in evaluating the true tumor response. This may be the reason for the non-significance of neutrophil count in this study, which is proven predictor and prognosticator in other studies. Third limitation is that the AUC and Youden's index are low in the ROC curve analysis of the post-CRT leukocyte, which may represent a low accuracy of the proposed cut-off value.

In conclusion, a post-CRT leukocyte count $\leq 3730 /$ L might be a useful prognostic factor to predict tumor response and increase survival in rectal cancer patients treated with preoperative CRT and curative surgery. It might be considered to reduce the adjuvant chemotherapy after surgery when the patients show a post-CRT leukocyte count $\leq 3730 / \mathrm{LL}$ and good tumor response with preoperative CRT for rectal cancer. Next, it might be interesting to examine the effects of supplementing 
with leukocytes when the leukocyte count decreases to less than the cut-off level during CRT and to assess whether this affects tumor response and patient survival.

\section{Conflict of Interest}

No potential conflict of interest relevant to this article was reported.

\section{References}

1. Shitara K, Matsuo K, Oze I, et al. Meta-analysis of neutropenia or leukopenia as a prognostic factor in patients with malignant disease undergoing chemotherapy. Cancer Chemother Pharmacol 2011;68:301-7.

2. Shitara K, Matsuo K, Takahari D, et al. Neutropenia as a prognostic factor in advanced gastric cancer patients undergoing second-line chemotherapy with weekly paclitaxel. Ann Oncol 2010;21:2403-9.

3. Tibaldi C, Vasile E, Bernardini I, Orlandini C, Andreuccetti M, Falcone A. Baseline elevated leukocyte count in peripheral blood is associated with poor survival in patients with advanced non-small cell lung cancer: a prognostic model. J Cancer Res Clin Oncol 2008;134:1143-9.

4. Poikonen $P$, Saarto $T$, Lundin J, Joensuu H, Blomqvist C. Leucocyte nadir as a marker for chemotherapy efficacy in node-positive breast cancer treated with adjuvant CMF. Br J Cancer 1999;80:1763-6.

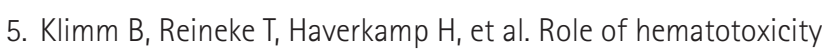
and sex in patients with Hodgkin's lymphoma: an analysis from the German Hodgkin Study Group. J Clin Oncol 2005;23:8003-11.

6. Choi CH, Kim WD, Lee SJ, Park WY. Clinical predictive factors of pathologic tumor response after preoperative chemoradiotherapy in rectal cancer. Radiat Oncol J 2012;30:99-107.

7. Shitara K, Matsuo K, Takahari D, et al. Neutropaenia as a prognostic factor in metastatic colorectal cancer patients undergoing chemotherapy with first-line FOLFOX. Eur J Cancer 2009;45:1757-63.

8. Lee SD, Park JW, Park KS, et al. Influence of anemia on tumor response to preoperative chemoradiotherapy for locally advanced rectal cancer. Int J Colorectal Dis 2009;24:1451-8.

9. Rades D, Kuhn H, Schultze J, et al. Prognostic factors affecting locally recurrent rectal cancer and clinical significance of hemoglobin. Int J Radiat Oncol Biol Phys 2008;70:1087-93.

10. Ding PR, An $X$, Zhang $R X$, et al. Elevated preoperative neutrophil to lymphocyte ratio predicts risk of recurrence following curative resection for stage IIA colon cancer. Int J Colorectal Dis 2010;25:1427-33.

11. Chiang SF, Hung HY, Tang R, et al. Can neutrophil-toIymphocyte ratio predict the survival of colorectal cancer patients who have received curative surgery electively? Int J Colorectal Dis 2012;27:1347-57.

12. Mandard AM, Dalibard F, Mandard JC, et al. Pathologic assessment of tumor regression after preoperative chemoradiotherapy of esophageal carcinoma: clinicopathologic correlations. Cancer 1994;73:2680-6.

13. Song JH, Kim SH, Lee JH, et al. Significance of histologic tumor grade in rectal cancer treated with preoperative chemoradiotherapy followed by curative surgery: a multi-institutional retrospective study. Radiother Oncol 2016;118:387-92.

14. Lee JH, Jang HS, Kim JG, et al. Prediction of pathologic staging with magnetic resonance imaging after preoperative chemoradiotherapy in rectal cancer: pooled analysis of KROG 10-01 and 11-02. Radiother Oncol 2014;113:18-23.

15. van de Schans SA, Janssen-Heijnen ML, Nijziel MR, Steyerberg $E W$, van Spronsen DJ. Validation, revision and extension of the Mantle Cell Lymphoma International Prognostic Index in a population-based setting. Haematologica 2010;95:1503-9.

16. Mabuchi S, Matsumoto $Y_{1}$ Isohashi $F_{\text {, et }}$ al. Pretreatment leukocytosis is an indicator of poor prognosis in patients with cervical cancer. Gynecol Oncol 2011;122:25-32.

17. Cho H, Kim JH. Multiplication of neutrophil and monocyte counts (MNM) as an easily obtainable tumour marker for cervical cancer. Biomarkers 2009;14:161-70.

18. Walsh SR, Cook EJ, Goulder F, Justin TA, Keeling NJ. Neutrophil-lymphocyte ratio as a prognostic factor in colorectal cancer. J Surg Oncol 2005;91:181-4.

19. Sanz GF, Sanz MA, Greenberg PL. Prognostic factors and scoring systems in myelodysplastic syndromes. Haematologica 1998;83:358-68.

20. Siddiqui M, Ristow K, Markovic SN, et al. Absolute lymphocyte count predicts overall survival in follicular lymphomas. $\mathrm{Br} J$ Haematol 2006;134:596-601.

21. Sasaki A, Iwashita Y, Shibata $K$, Matsumoto T, Ohta M, Kitano S. Prognostic value of preoperative peripheral blood monocyte count in patients with hepatocellular carcinoma. Surgery 2006;139:755-64.

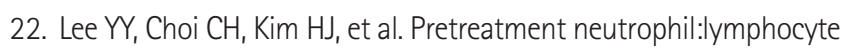
ratio as a prognostic factor in cervical carcinoma. Anticancer Res 2012;32:1555-61.

23. Saarto $T$, Blomqvist $C$, Rissanen $P$, Auvinen $A$, Elomaa I. 
Haematological toxicity: a marker of adjuvant chemotherapy efficacy in stage II and III breast cancer. Br J Cancer 1997;75:301-5.

24. Di Maio M, Gridelli C, Gallo C, et al. Chemotherapy-induced neutropenia and treatment efficacy in advanced non-smallcell lung cancer: a pooled analysis of three randomised trials. Lancet Oncol 2005;6:669-77.

25. Hirashima M, Higuchi S, Sakamoto K, Nishiyama T, Okada H. The ratio of neutrophils to lymphocytes and the phenotypes of neutrophils in patients with early gastric cancer. J Cancer Res Clin Oncol 1998;124:329-34.

26. Bais AG, Beckmann I, Lindemans J, et al. A shift to a peripheral
Th2-type cytokine pattern during the carcinogenesis of cervical cancer becomes manifest in CIN III lesions. J Clin Pathol 2005;58:1096-100.

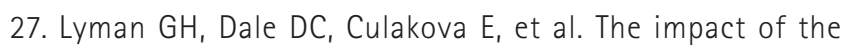
granulocyte colony-stimulating factor on chemotherapy dose intensity and cancer survival: a systematic review and meta-analysis of randomized controlled trials. Ann Oncol 2013;24:2475-84.

28. Aliper AM, Frieden-Korovkina VP, Buzdin A, Roumiantsev SA Zhavoronkov A. A role for G-CSF and GM-CSF in nonmyeloid cancers. Cancer Med 2014;3:737-46. 